\title{
Intelligent Model of Microwave Filter Using PDGS with Defected Rectangles
}

\author{
JIN Taobin ${ }^{[1,2]}$, JIN Jie ${ }^{2}$, YAO Ruipu ${ }^{1}$, LI Kejia ${ }^{2}$, ZHANG Yizhen ${ }^{1}$ \\ (1. School of Information Engineering, Tianjin University of Commerce, Tianjin ,300134, China.) \\ (2. School of Electronic Information Engineering, Tianjin University, Tianjin ,300072, China.) \\ E-mail: jintaobin@163.com
}

\begin{abstract}
Microwave filter is the kind of device which can separate different signals within a certain band of microwave frequency, widely used in microwave communication system. Periodic defected ground structures (PDGS) with defected rectangles have excellent filter properties when periodic unit amounts and structure sizes meet appropriate conditions. In this paper, intelligent model of PDGS with defected rectangles is developed for the first time on the basis of FDTD analysis. Periodic unit amounts, the structure sizes of PDGS with defected rectangles and the frequency are defined as the input samples of artificial neural network (ANN), transmission coefficient (S21) are defined as the output samples. Transmission coefficient of PDGS at any arbitrary periodic unit amounts, any arbitrary structure sizes and any arbitrary frequency within training values range can be obtained quickly from intelligent model after the ANN has been successfully trained with the Bayesian Regularization algorithm. Finally, intelligent model has been approved by FDTD results. It is also showed that intelligent model is very effective, which will provide powerful approach for the precise analysis and quick design of microwave filter using PDGS with defected rectangles.
\end{abstract}

Keywords-microwave filter; periodic unit amounts; Bayesian Regularization algorithm; transmission coefficient

\section{INTRODUCTION}

Defected ground structures (DGS) are recently one of the hottest topics which are researched in microwave domain, which developed from the photonics bandgap (PBG) structures. DGS can achieve high-performance which can not be obtained by conventional technology. Because of the advantage of DGS, such as having small structure size and simple model, it has been widely used in the design of microwave filter、oscillator, coupler, power amplifier and antenna. PDGS with defected rectangles are the structures that are etched periodically using the rectangular holes on their ground plane. Rectangular defected areas and one connecting slot correspond to the equivalently added inductance (L) and capacitance (C), respectively. Accordingly, a resonance occurs at a certain frequency because of the parallel L-C circuit. Inversely, it is intuitively known that the equivalent circuit includes a pair of parallel inductor-capacitor from the resonant phenomenon in the S-parameter. This means that the microstrip structure having PDGS with defected rectangles does not have all-pass characteristics, but restricted passband properties. PDGS with defected rectangles can be used to prohibit the propagation of electromagnetic waves within a certain band of frequency. Broadband filter can be implemented through the cascading of many PDGS with defected rectangles, which have wide application prospects in microwave circuits and antenna domain.

Finite-difference time-domain (FDTD) method is frequently utilized to analyze PDGS, which is a kind of electromagnetic numerical value analysis methods that are precise and strict. FDTD has a strong ability of simulating many complex structures, and can gain time domain waveform of the subject investigated ${ }^{[1 \sim 3]}$. Frequency characteristic can be correspondingly obtained by Fourier transform, furthermore it can avoid sick results likely occurring in the process of calculating equations, and decrease largely memory space and computing time, so FDTD method is applied to research PDGS. FDTD with the perfectly matched layer (PML) absorbed boundary is used to analyze PDGS because it has an advantage over traditional Mur absorbed boundary, such as more simple feedback model, smaller calculation space, faster convergence, unconstrained by angle of incidence and frequency. FDTD is precise and strict, but the time-consuming disadvantage of FDTD seriously hinders the precise analysis and quick design of PDGS. Effective models of PDGS are necessarily built in order to facilitate computer aided design and save time. In this paper, intelligent model of PDGS with defected rectangles is developed for the first time on the basis of FDTD analysis. Periodic unit amounts, the structure sizes of PDGS with defected rectangles and the frequency are defined as the input samples of the ANN, transmission coefficient (S21) are defined as the output samples. Transmission coefficient of PDGS at any arbitrary periodic unit amounts, any arbitrary structure sizes and any arbitrary frequency within training values range can be obtained quickly from intelligent model after the ANN has been successfully trained with the Bayesian Regularization algorithm. Although training ANN will spend much time, trained intelligent model can quickly give precise results which have finally been approved by FDTD results ${ }^{[4 \sim 6]}$. It is also showed that intelligent model is very effective, which will provide powerful approach for the precise analysis and quick design of microwave filter using PDGS with defected rectangles.

\section{PDGS WITH DEFECTED RECTANGLES}

The structures are shown in Fig.1, in which defected rectangles are periodic unit, $l_{1}$ is the length of the whole structures, $l_{2}$ is the width of the whole structures, $w$ is the width of microstrip line, $d$ is the space between periodic unit, a and $b$ are the structure sizes. PDGS with defected rectangles shown in Fig.1 have obvious filter characteristic after being 
analyzed through FDTD (defected rectangles being supposed equal intervals distribution).

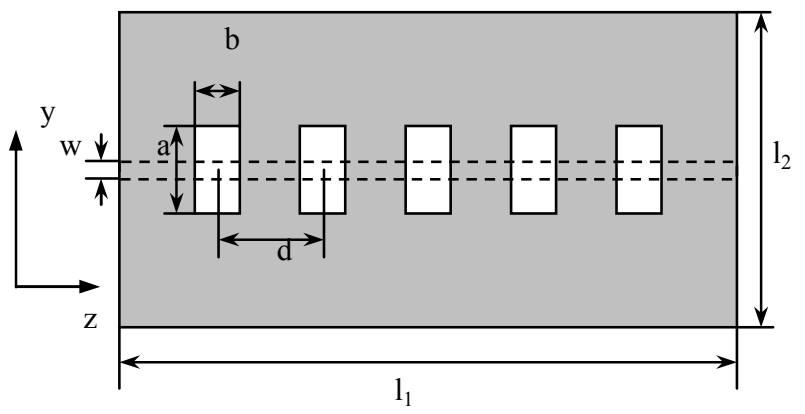

Figure 1. The structure of one dimension PDGS

After one dimension distribution PDGS whose periodic unit being defected rectangles as shown in Fig.1 are analyzed with FDTD, the whole structure size is $1_{1}=120 \mathrm{~mm}, l_{2}=30 \mathrm{~mm}$, the width of microstrip line is $\mathrm{w}=3 \mathrm{~mm}$, the space between periodic unit is $d=20 \mathrm{~mm}$, the relative dielectric constant is $\varepsilon r$ $=2.65$, the thickness of dielectric slab is $1 \mathrm{~mm}$. Periodic units amounts $n$ are 5, 4, 3, 2, 1 respectively. Defected rectangular holes have the same structure sizes between $6 \mathrm{~mm}$ and $10 \mathrm{~mm}$, that is to say, values range of the structure sizes $a$ and $b$ is from $6 \mathrm{~mm}$ to $10 \mathrm{~mm}$.

\section{BAYESIAN REGULARIZATION ALGORITHM}

Levenberg-Marquardt BP is the transformative BP neural networks algorithm based on standard numerical optimization techniques. Learning time can be shortened using L-M optimization, which is appropriate for training neural networks and has better results in practical application. The process is simplified to be written in the references. In the neural networks training process, an important problem is to avoid over-fitting neural networks in order to improve the generalization ability. In this paper the BP neural networks model has been trained with the Bayesian Regularization algorithm based on the L-M optimization theory. The values of the weight and threshold quantity can be optimized. Bayesian Regularization algorithm can improve the generalization ability of the networks, the establishment of training course for output error of upper layer and the values of the weight and threshold quantity. The special performance function is utilized to adjust the values of the weight and threshold quantity to minimize the performance function ${ }^{[7]}$.

Before and after the adjustment, the error functions are mse and msereg.

$$
\begin{aligned}
\text { mse } & =\frac{1}{N} \sum_{i=1}^{N}\left(e_{i}\right)^{2} \\
\text { msereg } & =\gamma \cdot m s e+(1-\gamma) \cdot m s w
\end{aligned}
$$

In which $e_{i}$ is networks error, $\gamma$ is error ratio of adjustment function parameters, $m s w$ is neural networks and the mean-square value for the values of the weight and threshold quantity.

$$
m s w=\frac{1}{n} \sum_{j=1}^{n} w_{j}^{2}
$$

Bayesian Regularization algorithm will be utilized to enable output response smoothen and to enhance the generalization ability of the networks to avoid over-fitting.

\section{INTELLIGENT MODEL OF PDGS}

Intelligent model of PDGS with defected rectangles is developed for the first time through ANN theory. The precise transmission coefficient (S21) of PDGS is obtained on the basis of FDTD analysis. Periodic unit amounts n, the structure sizes $\mathrm{a}, \mathrm{b}$ and the frequency $\mathrm{f}$ are defined as the input samples of the ANN model, the parameters of transmission coefficient are defined as the output samples. The values range of structure sizes $a, b$ is $6 \mathrm{~mm} \leq \mathrm{a} \leq 10 \mathrm{~mm}, \quad 6 \mathrm{~mm} \leq \mathrm{b} \leq 10 \mathrm{~mm}$. The range of periodic unit amounts $n$ is $1 \leq n \leq 5 \quad(n=1,2,3,4,5)$. Through analyzing transmission characteristic of PDGS with defected rectangles, it is obvious that they have excellent filter characteristic within the frequency band $3 \sim 7 \mathrm{GHz}$, nevertheless their filter characteristic is flat comparatively without the frequency band $3 \sim 7 \mathrm{GHz}$. The values range of frequency $\mathrm{f}$ is limited $2.8 \mathrm{GHz} \leq \mathrm{a} \leq 8 \mathrm{GHz}$. In the values range of these parameters which are $n, a, b$ and $f$, transmission coefficient (S21) is calculated by FDTD. The samples collected will be divided into training samples and test samples. Intelligent models are trained arriving at accuracy set in advance. The accuracy set in advance is higher, and the trained neural networks are more precise. Of course training time spent will be much longer.

Transmission coefficient (S21) of PDGS at any arbitrary parameters (including periodic unit amounts $n$, structure sizes $a, b$, frequency f) within training values range can be obtained quickly from intelligent model after the ANN has been successfully trained with the Bayesian Regularization algorithm. As the model has been trained with the Bayesian Regularization algorithm, transmission coefficient (S21) of PDGS at any arbitrary parameters $(n, a, b)$ being not trained in training values range (for example $n=1, a=6 \mathrm{~mm}, b=8 \mathrm{~mm} ; n=4$, $\mathrm{a}=8 \mathrm{~mm}, \mathrm{~b}=9 \mathrm{~mm}$ ) and the frequencies can be obtained quickly from intelligent model, which greatly overcomes the timeconsuming disadvantage of FDTD. Although training ANN will spend much time, trained intelligent model can quickly give precise results which have finally been approved by FDTD results. Finally, intelligent model has been approved by FDTD results. The results of intelligent model and the calculated results of FDTD are compared with in Fig.2 Fig.6. It is shown that these results match very well each other, the average absolute error is less than $2 \%$, which will provide powerful approach for the precise analysis and quick design of microwave filter using PDGS with defected rectangles. 


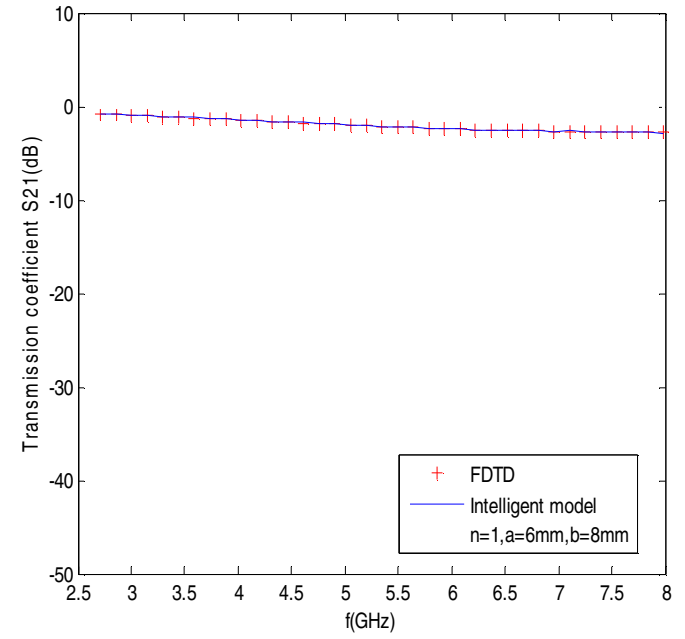

Figure 2. The calculated results of intelligent model and FDTD

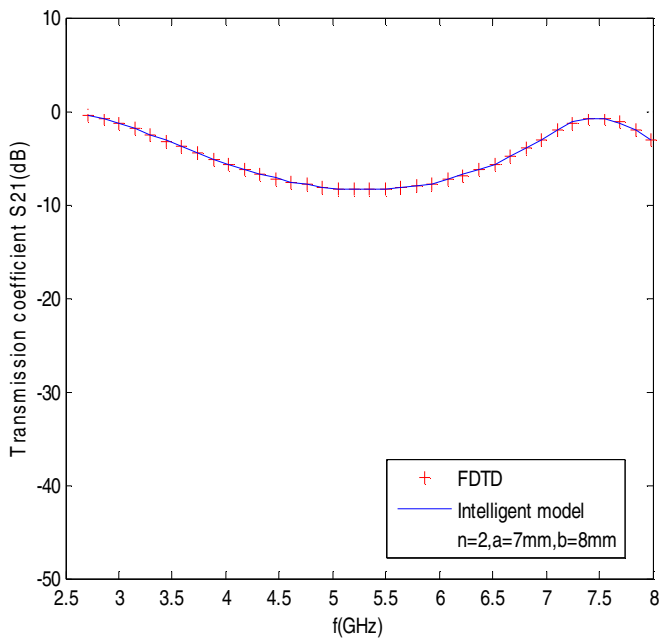

Figure 3. The calculated results of intelligent model and FDTD

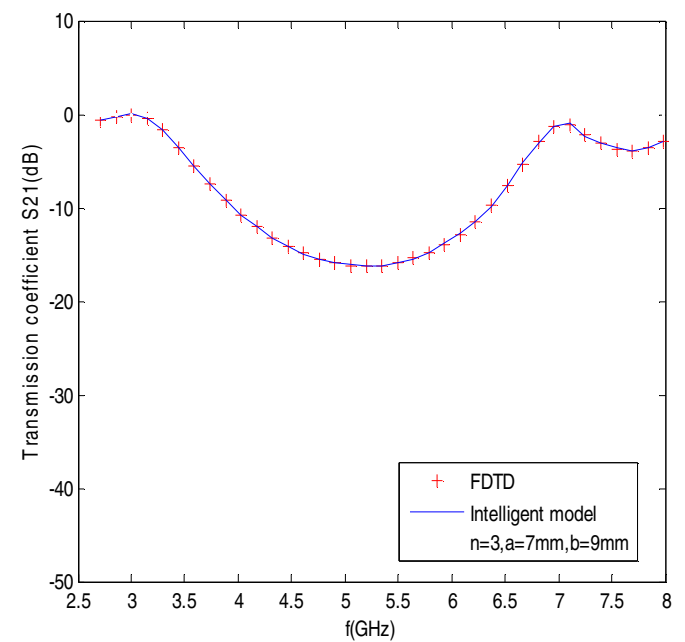

Figure 4. The calculated results of intelligent model and FDTD

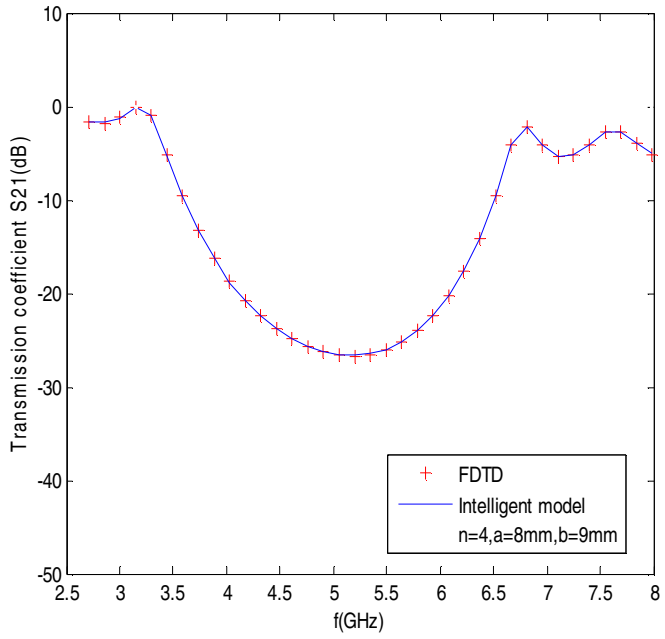

Figure 5. The calculated results of intelligent model and FDTD

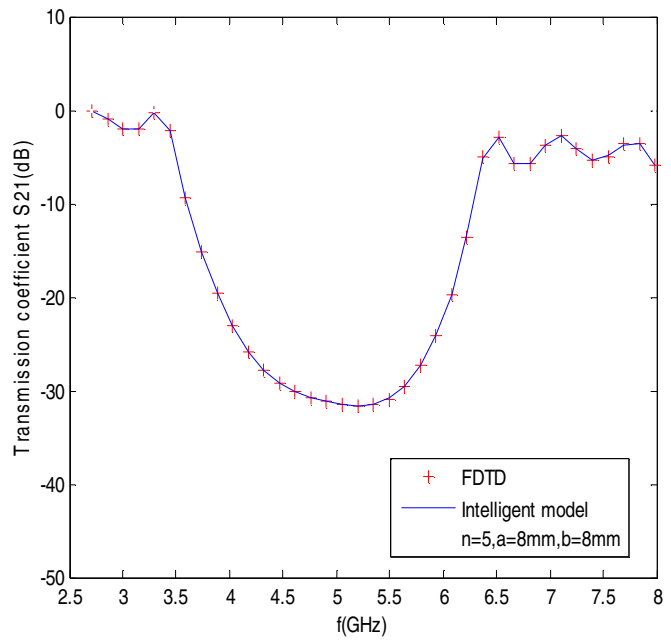

Figure 6. The calculated results of intelligent model and FDTD

\section{CONCLUSIONS}

Intelligent model are developed for PDGS with defected rectangles for the first time in this paper, and the calculated results of intelligent model are agreement with the results of FDTD. It is shown that intelligent model is very precise and effective. Although training ANN model has spent much time, the successful intelligent model can quickly provide precise answers to the task in their training values range. It is also showed that intelligent model is very effective, which can seriously overcome the time-consuming disadvantage of FDTD to facilitate the precise analysis and quick design of microwave filter using PDGS with defected rectangles.

\section{ACKNOWLEDGMENT}

This paper is supported by National Science Foundation of China (No.60777014). 


\section{REFERENCES}

[1] Jun-Seok Park,Jun-Sik Yun, Dal Ahn. A Design of the Novel CoupledLine Bandpass Filter Using Defected Ground Structure with Wide Stopband Performance [J]. IEEE Trans. MTT., 2002, 50(9) : 2037 2043.

[2] Dal Ahn,Chul-Soo Kim,Juno Kim,etc.. A design of low-pass filter using the novel microstrip defected ground structure [J]. IEEE Trans. MTT. 2001, 49 (1) : 86 93.

[3] Jinping Yang, Wen Wu. Compact Elliptic-Function Low-Pass Filter Using Defected Ground Structure [J]. IEEE Microwave and Wireless Components Letters, 2008,18(9) : 578 580.

[4] LI Yuan,LI Huancai,DING Ronglin. Comparison of Characteristics of Periodic and Non-Periodic Defected Ground Structures [J]. Transactions of Tianjin University,2006,12(5) : 387 390.

[5] Jun-Seok Park, Myeong-Sub Jung . A Novel Defected Ground Structure for an Active Device Mounting and Its Application to a Microwave Oscillator [J]. IEEE Microwave and Wireless Components Letters, 2004,14(5) : 198 200.

[6] Mrinal K M, Subrata Sanyal. A Novel Defected Ground Structure for Planar Circuits[J]. IEEE Microwave and Wireless Components Letters, 2006,16(2) : 93 95.

[7] Wang Fang,Devabhaktuni V K,Zhang Q J. A hierarchical neural network approach to the development of a library of neural models for microwave design [J]. IEEE Trans. MTT. , 1998,46(12) : 2391 2403. 